\title{
ON THE ANALYSIS OF PHENYLTHIOHYDANTOIN AMINO ACIDS BY HIGH PERFORMANCE LIQUID CHROMATOGRAPHY
}

\author{
by \\ SVEN ERIK GODTFREDSEN and RONALD W. A. OLIVER" \\ Department of Chemistry, Carlsberg Laboratory, \\ Gamle Carlsberg Vej 10, DK-2500 Copenhagen Valby \\ 1)Present address: \\ Department of Biochemistry, University of Salford. \\ Salford 5, Lancs., U.K.
}

Keywords: Phenylthiohydantoin amino acids, high performance liquid chromatography, high speed liquid chromatography, micro-bore columns

\begin{abstract}
The key experimental variables and the corresponding separations of synthetic mixtures of phenylthiohydantoin amino acids achieved by high performance liquid chromatography have been abstracted from the literature and are listed in tabular form. From this table it is seen that the large majority of separations reported since 1977 have been performed utilizing stainless steel tubing of large internal diameter (i.d. $\geqslant 3.9 \mathrm{~mm}$ ). The reason for the widespread adoption of this particular practice is that a number of earlier studies have indicated that use of small diameter columns leads to significant loss of chromatographic resolution. An experimental study, employing two commercial chomatographic systems and tubings of differing internal diameter (i.d. 4.5 to 1.5 $\mathrm{mm}$ ), leads to the conclusion that columns of reverse phase support material packed in stainless steel micro-bore tubing (i.d. $\leqslant 2 \mathrm{~mm}$ ) can effect separation of phenylthiohydantoin amino acids with similar efficiency as larger diameter columns. The practical advantages of employing micro-bore columns in general are discussed and adoption of micro-bore columns for the routine separation of this particularly important group of amino acid derivatives is recommended.
\end{abstract}

\footnotetext{
Abbreviations: HPLC $=$ high performance liquid chromatography, $\mathrm{PTH}=$ phenylthiohydantoin, $\mathrm{PC}=$ paper chromatography, TLC = thin layer chromatography, GLC = gas liquid chromatography, IEC $=$ ion exchange chromatography, s.s. = stainless steel, i.d. = internal diameter.
} 


\section{INTRODUCTION}

Methods for the identification of PTH-amino acids obtained by degradation of peptides and proteins according to the procedure developed by EDMAN (2) are of considerable importance since elucidation of the primary structure of peptides and proteins rely almost exclusively on application of the Edman procedure. Accordingly, a wide variety of chromatographic techniques, such as paper chromatography, thin layer chromatography, gas liquid chromatography and ion exchange chromatography have been adapted to this important analytical problem with the aim of providing unambigous, rapid, reproducible, sensitive and economical methods for the analysis of PTH-amino acids and closely related amino acid derivatives (for a review, see ref. 6). The developments in liquid chromatography in the 1960's have led to the modern practice of high performance liquid chromatography which would be expected to be particularly well suited to fulfil these analytical requirements. Thus, PTH-amino acids being UV absorbing at 254 $\mathrm{nm}$ are amenable to detection and accurate quantitation by UV detector systems commonly used in most HPLC equipment. Moreover, identification of the individual components in a mixture of the amino acid derivatives can be made by reference to the chromatographic behaviour of authentic specimens of PTH-amino acids, and, furthermore, it is reasonable to expect that the time required for analysis of a mixture of PTH-amino acids would be comparable to the 20 minute time period necessary to handle the daily volume of samples generated by the currently available automated peptide sequencers.

The extent to which these expectations have been realized in practice can be demonstrated by reference to Table I which lists the HPLC separations of PTH-amino acids published since 1973. A detailed study of this table leads to the following observations:

1. The majority (28) of the 39 separations reported in the 35 papers abstracted have been performed using columns of reverse phase support material. Only seven separations (paper $1,3,5,7,8,9$, and 21$)$ were performed on silica support material whilst three papers (nos. 18, 19 and 23) report separations on non-commercial tripeptides bonded to silica supports.
2. One paper (No 21) reports an efficient separation on a silica support. However, this method has not been widely adopted probably because of the poor reproducibility of separations obtained using this support.

3. The whole range of commercial reverse phase supports have been employed. The following list details the number of the reported applications of each of the hydrocarbon support materials:

$\mu$ Bondapak C18 Paper Nos. 8, 10, 14, 16, 22, $25,27,28,30,33,34$

Corasil C18 Paper Nos. 2, 4, 6

Spherisorb ODS Paper Nos. 31, 35

Partisil ODS C18 Paper No. 12

ODS $-18 \quad$ Paper No. 26

Zorbax ODS Paper Nos. 11, 17, 20, 24

Lichrosorb C8 Paper Nos. 29, 32

Lichrosorb C18 Paper No. 13

Hypersil ODS Paper No. 31

In addition, two papers (Nos. 6 and 11) report separations involving bonded ether phases, Permaphase ETH, and one paper (No. 15) reports the use of Micropak CN.

4. Whilst hydrocarbon $\mathrm{C} 18$ and $\mathrm{C} 8$ bonded support material has been used for 25 of the 28 separations on reverse phase columns, no detailed comparative studies of the efficiences of the separations obtained with the various commercial materials utilising these particular bonded phases has yet been published. A detailed comparison of the separations abstracted in Table I leads to the conclusion that reproducible, rapid and efficient separation of mixtures of PTH-amino acids can be carried out on any of the commercially available $\mathrm{C} 18$ or $\mathrm{C} 8$ reverse phase support materials packed in stainless steel columns of diameters $\geqslant 3.9 \mathrm{~mm}$ and used in conjunction with gradient elution involving mixtures of aqueous sodium acetate buffers and either acetonitrile or methanol at elevated temperatures.

5. Since 1977 all separations reported to be carried out on reverse phase support material packed in stainless steel tubing employed wide bore tubing (i.d. $\geq 3.9 \mathrm{~mm}$ ). One paper (No. 26) published since 1977 reports the use of a small diameter column which, however, was packed in teflon tubing (i.d. $0.5 \mathrm{~mm}$ ).

The value of collating chromatographic separations of a complex mixture lies in the 
Table I

Summary of the experimental conditions and separations reported for synthetic mixtures of phenylthiohydantoin amino acids by high performance liquid chromatography.

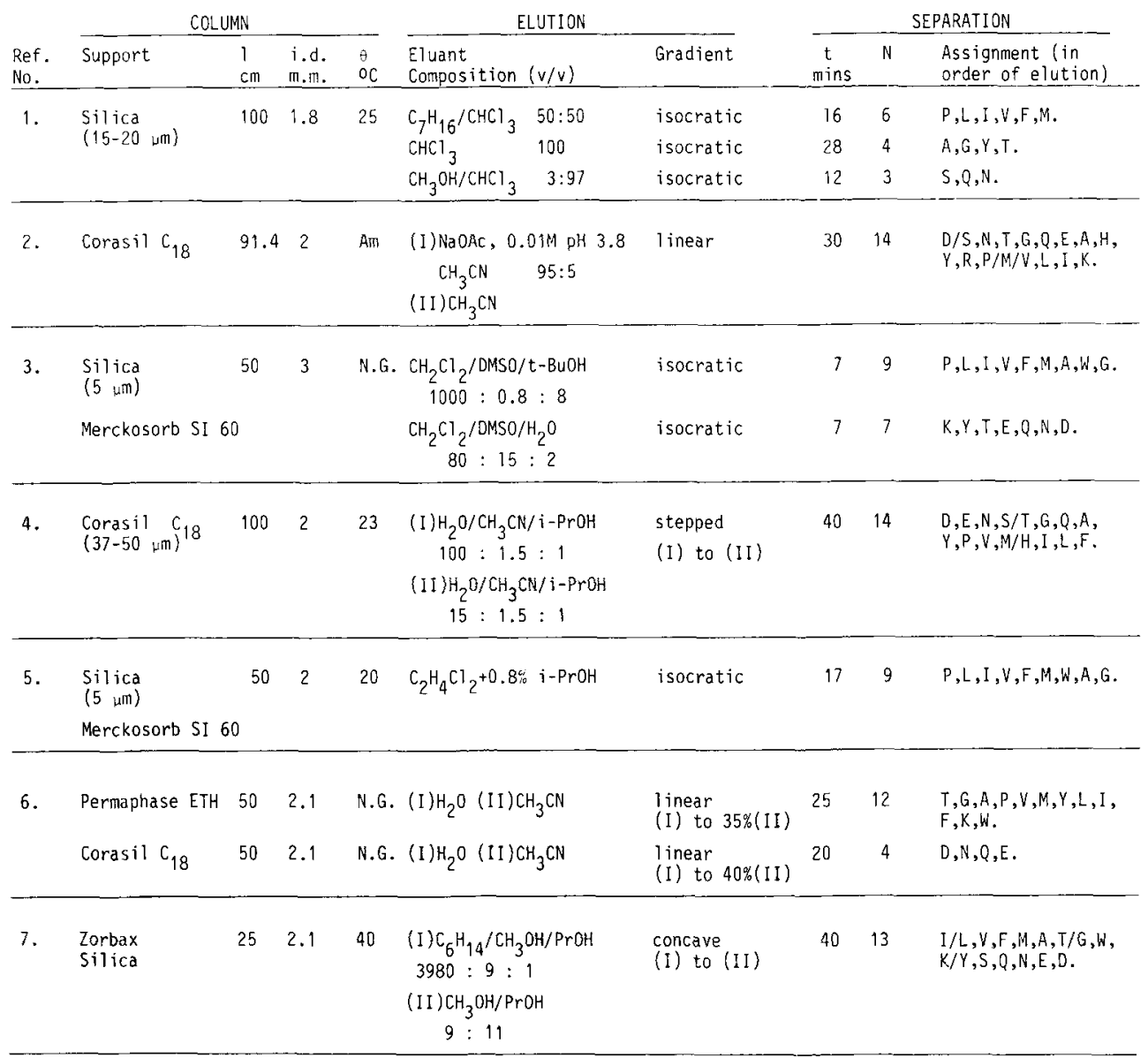

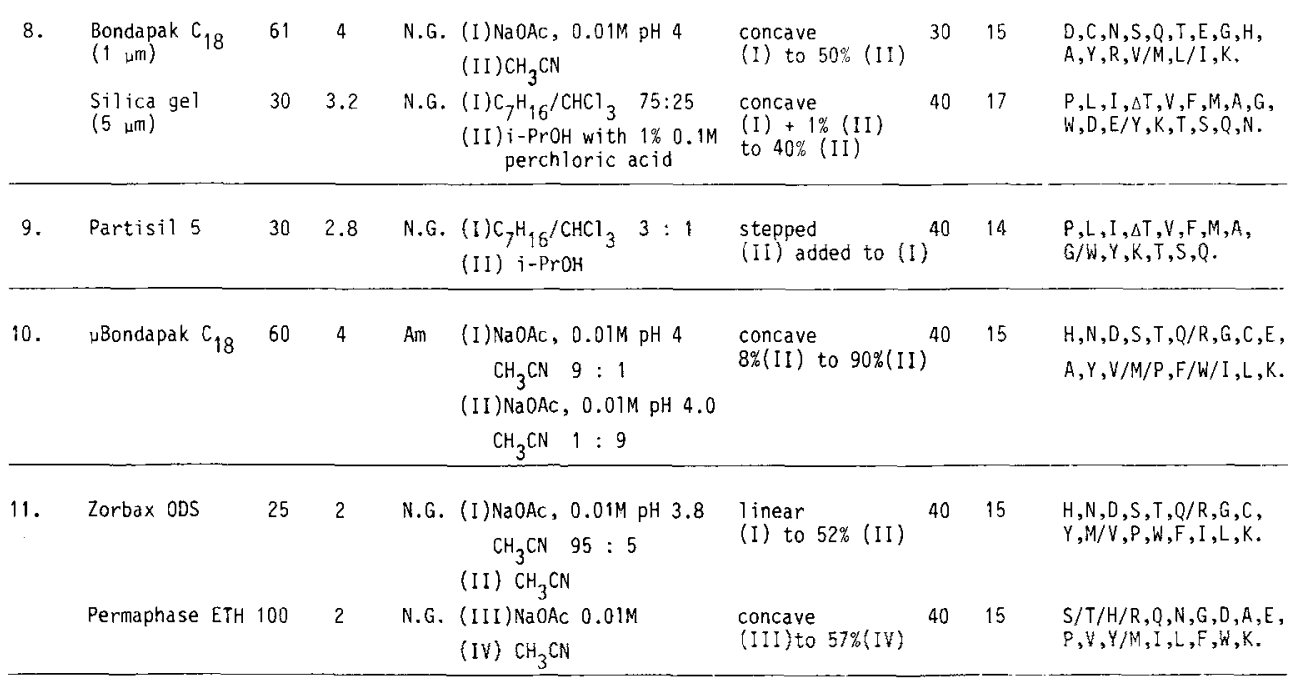


S. E. Godtfredsen \& R. W. A. Oliver: HPLC of PTH-amino acids

\begin{tabular}{|c|c|c|c|c|c|c|c|c|c|}
\hline \multirow[b]{2}{*}{$\begin{array}{l}\text { Ref. } \\
\text { No. }\end{array}$} & \multicolumn{4}{|c|}{ COLUMN } & \multicolumn{2}{|l|}{ ELUTION } & \multicolumn{3}{|c|}{ SEPARATION } \\
\hline & Support & $\begin{array}{l}1 \\
\mathrm{~cm}\end{array}$ & $\begin{array}{l}\text { i.d. } \\
\text { m.m. }\end{array}$ & $\stackrel{\theta}{\circ}^{\circ} \mathrm{C}$ & $\begin{array}{l}\text { Eluant } \\
\text { Composition }(v / v)\end{array}$ & Gradient & $\stackrel{t}{\mathrm{t}}$ & N & $\begin{array}{l}\text { Assignment (in } \\
\text { order of elution) }\end{array}$ \\
\hline \multirow[t]{3}{*}{12.} & $\begin{array}{l}\text { Partisil oDS } \\
\mathrm{C}_{18}(10 \mu \mathrm{m})\end{array}$ & 25 & 4.6 & & $\begin{array}{l}\text { (I) } \mathrm{NaOAC} 0.01 \mathrm{M} \mathrm{pH} 4 \\
10 \% \mathrm{CH}_{3} \mathrm{CN}\end{array}$ & isocratic & 6 & 8 & $C, D, N, T, S, G, E, Q$. \\
\hline & & & & & $\begin{array}{l}\text { (11) } \mathrm{NaOAC} 0.01 \mathrm{M} \mathrm{pH} 4 \\
20 \% \mathrm{CH}_{3} \mathrm{CN}\end{array}$ & isocratic & 8 & 4 & $A, Y, V, M$ \\
\hline & & & & & $\begin{array}{l}\text { (III) } \mathrm{NaOAC}_{2} 0.01 \mathrm{M} \mathrm{pH} 4 \\
30 \% \mathrm{CH}_{3} \mathrm{CN}\end{array}$ & isocratic & 11 & 8 & $P, W, I, L, F, K, H, R$. \\
\hline 13. & Lichrosorb $C_{18}$ & 25 & 4.6 & Am & $\begin{array}{l}\text { (I) } \mathrm{NaOAC} 0.01 \mathrm{M} \text { pH } 4.6 \\
\text { (II) } \mathrm{CH}_{3} \mathrm{CN}\end{array}$ & $\begin{array}{l}1 \text { inear } \\
\text { from } 5 \% \text { (II) to } \\
40 \% \text { (II) }\end{array}$ & 25 & 11 & $\begin{array}{l}D, N, E, Q, G, A, Y, \\
V / M / P, I / F / W, L, K .\end{array}$ \\
\hline 14. & $\mu$ Bondapak $C_{18}$ & 60 & 4 & N.G. & $\begin{array}{l}\text { (1) } \mathrm{NaOAC} \quad 0.01 \mathrm{M} \mathrm{pH} 4 \\
\mathrm{CH}_{3} \mathrm{CN} \quad 95: 5 \\
\text { (II) } \mathrm{NaOAC} \quad 0.01 \mathrm{M} \quad \mathrm{pH} 4\end{array}$ & $\begin{array}{l}l \text { inear } \\
\text { from } 5 \% \text { (II) to } \\
60 \% \text { (II) }\end{array}$ & 60 & 12 & $\begin{array}{l}D, N, S, T, Q, G, E, A, Y, \\
P, L, K .\end{array}$ \\
\hline & & & & & $\mathrm{aH}_{3} \mathrm{CN} \quad 5: 95$ & & & & \\
\hline 15. & $\begin{array}{l}\text { Micropak CN } \\
(10 \mu \mathrm{m})\end{array}$ & 25 & 2.1 & N.G. & $\begin{array}{l}\text { (I) } \mathrm{C}_{6} \mathrm{H}_{14} \\
\text { (II) } \mathrm{CH}_{2} \mathrm{Cl}_{2} \text {, i-PrOH } 8: 2\end{array}$ & $\begin{array}{l}\text { mixed linear } \\
\text { and isocratic } \\
\text { from (I) to (II) }\end{array}$ & 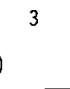 & 16 & $\begin{array}{l}P, L / I, V, F, M, A, T, G, \\
Y, K, S, W, Q, N, E, D .\end{array}$ \\
\hline 16 & u8ondapak $\mathrm{C}_{18}$ & N.G. & N.G. & N.G. & $\begin{array}{l}\text { (1) } \mathrm{H}_{2} \mathrm{O}, \mathrm{CH}_{3} \mathrm{OH}, \mathrm{HOAC} \\
\mathrm{pH} 4.1 \quad 90: 10: 0.25 \\
\text { (1I) } \mathrm{H}_{2} \mathrm{O}, \mathrm{CH}_{3} \mathrm{OH}, \mathrm{HOAC} \\
10: 90: 0.025\end{array}$ & $\begin{array}{l}\text { linear } \\
95 \%(I)+5 \%(I I) \\
\text { to } \\
40 \%(I)+60 \%(I I)\end{array}$ & N.G. & N.G. & N.G. \\
\hline \multirow[t]{3}{*}{17.} & Zorbax ODS & 25 & 4.6 & 62 & $\begin{array}{l}\text { (I) } \mathrm{NaOAC} 0.01 \mathrm{MPH} 4.5 \\
\text { (II) } \mathrm{CH}_{3} \mathrm{CN}\end{array}$ & $\begin{array}{l}\text { mixed linear } \\
\text { and isocratic } \\
24 \% \text { (II) to } \\
45 \% \text { (II) }\end{array}$ & 20 & 19 & $\begin{array}{l}D, E, N, S, Q, T, G, A, Y, H, \\
V, M, P, W, K, F, I, L, R .\end{array}$ \\
\hline & & & & & $\mathrm{NaOAC} \quad 0.01 \mathrm{M}$ pH 4.5 & isocratic & 13 & 19 & $\begin{array}{l}\text { As above except } S / Q \\
\text { and } P \text { follows } W\end{array}$ \\
\hline & & & & & $\mathrm{CH}_{3} \mathrm{CN} \quad 58: 42$ & & & & \\
\hline 18. & $\begin{array}{l}\text { Bonded tripep- } \\
\text { tide L-Va7- } \\
\text { L-Ala-L-Ser } \\
\text { to } \mathrm{SiO}_{2} \text { gel CT } \\
\end{array}$ & 25 & 3.8 & 20 & $\begin{array}{l}1 \% \text { citric acid-water } \\
\text { pH } 2.5\end{array}$ & isocratic & 70 & $18^{*}$ & $\begin{array}{l}H, R, S, T, N, G, Q, A, E, \\
D, Y, M, P, Y, L, I, F, W .\end{array}$ \\
\hline 19. & $\begin{array}{l}\text { Bonded tripep- } \\
\text { tide L-Val- } \\
\text { L-Phe-L-Val } \\
\text { to Partisil } 10\end{array}$ & 25 & 2.1 & N.G. & $\begin{array}{l}1 \% \text { citric acid-water } \\
\mathrm{pH} 2.5\end{array}$ & isocratic & N.G. & $18^{\star}$ & $\begin{array}{l}H, R, T, S, N, Q, G, A, E, \\
V, P, D, M, Y, I, L, F, W .\end{array}$ \\
\hline 20. & Zorbax ODS & 25 & 4.6 & 62 & 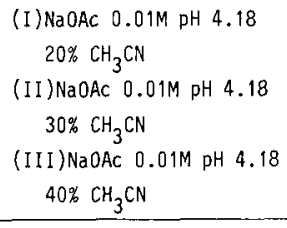 & $\begin{array}{l}\text { three step } \\
\text { isocratic } \\
\text { programme }\end{array}$ & 25 & 14 & $\begin{array}{l}D, N, E / S, Q, T, G, A, Y, \\
M, V, P / W, F / K, I, L .\end{array}$ \\
\hline 21. & uPorasil & 30 & 3.9 & N.G. & $\begin{array}{l}\text { (I) } \mathrm{i}-\mathrm{C}_{8} \mathrm{H}_{18} \\
\text { (II) } \mathrm{CH}_{3} \mathrm{OH}, \mathrm{i}-\mathrm{PrOH} 9: 11 \\
\text { (III) } \mathrm{i}-\mathrm{C}_{8} \mathrm{H}_{18}\end{array}$ & $\begin{array}{l}\text { concave } \\
5 \%(\text { II) to } \\
70 \% \text { (II) in (I) }\end{array}$ & 25 & 14 & $\begin{array}{l}L / I, V, P, F / M, A, G, T, \\
W, S, Y, K, N, Q, D / E .\end{array}$ \\
\hline & & & & & $\begin{array}{l}\mathrm{CHCl}_{3} 7: 3 \\
\text { (IV) } \mathrm{i}_{8} \mathrm{H}_{18} \\
\mathrm{CH}_{3} \mathrm{OH}_{3} \mathrm{CHCl}_{3} \\
15: 42.5: 42.5\end{array}$ & $\begin{array}{l}\text { concave } \\
1 \% \text { (IV) to } \\
100 \% \text { (IV) in (III }\end{array}$ & $\begin{array}{l}25 \\
\text { I) }\end{array}$ & 15 & $\begin{array}{l}P, L, I, V, F, M, A, G, W / K, \\
Y, T, S, P / E, Q, N .\end{array}$ \\
\hline
\end{tabular}


S. E. Godtfredsen \& R. W. A. Oliver: HPLC of PTH-amino acids

\begin{tabular}{|c|c|c|c|c|c|c|c|c|c|}
\hline \multirow[b]{2}{*}{$\begin{array}{l}\text { Ref. } \\
\text { No. }\end{array}$} & \multicolumn{4}{|c|}{ COLUMN } & \multicolumn{2}{|l|}{ ELUTION } & \multicolumn{3}{|c|}{ SEPARATION } \\
\hline & Support & $\begin{array}{l}1 \\
\mathrm{~cm}\end{array}$ & $\begin{array}{l}\text { i.d. } \\
\text { m.m. }\end{array}$ & $\begin{array}{l}\theta \\
\circ C\end{array}$ & $\begin{array}{l}\text { Eluant } \\
\text { Composition }(\mathrm{v} / \mathrm{v})\end{array}$ & Gradient & mins & N & $\begin{array}{l}\text { Assignment (in } \\
\text { order of elution) }\end{array}$ \\
\hline 22. & uBondapak $C_{18}$ & 30 & 3.9 & & 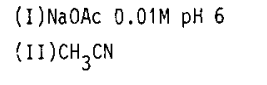 & $\begin{array}{l}\text { exponential } \\
\text { from } 21 \%(I I) \text { to } \\
38 \%(I I)\end{array}$ & 20 & 15 & $\begin{array}{l}D, E, C, N, S, T, Q / H, G, R, \\
A, Y, V / P / M, I / F, L / W, K .\end{array}$ \\
\hline 23. & $\begin{array}{l}\text { Bonded tripep- } \\
\text { tide L-Val- } \\
\text { L-Ala-L-Pro } \\
\text { to Partisil } 10\end{array}$ & 30 & 2.1 & N.G. & $\begin{array}{l}1 \% \text { citric acid-water } \\
\text { pH } 2.5\end{array}$ & isocratic & 40 & 9 & $H, T, G, E, V, P, Y, F, W$. \\
\hline 24. & Zorbax ODS & 25 & 4.6 & 55 & $\begin{array}{l}\text { (I) } \mathrm{NaOAC} \quad 0.02 \mathrm{M} \mathrm{pH} 5 \\
\text { (II) } \mathrm{CH}_{3} \mathrm{CN}\end{array}$ & $\begin{array}{l}\text { multi linear } \\
\text { isocratic } \\
\text { (I) to } 90 \% \text { (II) }\end{array}$ & 20 & 20 & $\begin{array}{l}D, E, N, S, Q, T, G, H / S, \\
A, Y, C, M, V, P, W, K, F, \\
I, L, R .\end{array}$ \\
\hline 25. & «Bondapak $C_{18}$ & 30 & 3.9 & 26 & $\begin{array}{l}\text { (I) } \mathrm{NaOAC} 0.005 \mathrm{M} \text { pH } 5.3 \\
23 \% \mathrm{CH}_{3} \mathrm{OH} \\
20 \mu 1 \text { acetone } \\
\text { (II) } \mathrm{CH}_{3} \mathrm{OH} \text {-water } 44: 56\end{array}$ & $\begin{array}{l}\text { mixed } \\
\text { exponential } \\
\text { isocratic } \\
\text { programme } \\
\text { (I) to (II) }\end{array}$ & 16 & 17. & $\begin{array}{l}D, E, N, S, T, G, Q, \Delta T, A, \\
Y, P, V, W, F, I, L, K .\end{array}$ \\
\hline 26. & $\begin{array}{l}\text { ODS-18 } \\
(5 \mu \mathrm{m})\end{array}$ & 9 & 0.5 & N.G. & $\begin{array}{l}\text { (I) } \mathrm{NaOAC} 0.005 \mathrm{M} \mathrm{pH} 5.3 \\
23 \% \mathrm{CH}_{3} \mathrm{OH} \\
\text { (II) } \mathrm{H}_{2} \mathrm{O}, \mathrm{CH}_{3} \mathrm{CN}, \mathrm{i}-\mathrm{PrOH} \\
7.5: 1.5: 1\end{array}$ & $\begin{array}{l}\text { isocratic } \\
\text { isocratic }\end{array}$ & $\begin{array}{l}25 \\
40\end{array}$ & 7 & $\begin{array}{l}D, E, N, S, T, G, Q . \\
A, Y, P / M / N, W, I / L / F, K .\end{array}$ \\
\hline 27. & $\mu$ Bondapak & 30 & 4 & $\begin{array}{l}20- \\
23\end{array}$ & 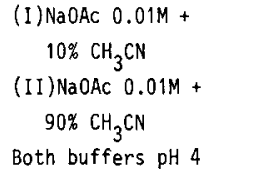 & $\begin{array}{l}25 \% \text { (II) to } \\
50 \% \text { (II) } \\
2 \% \text { (II) to } \\
30 \% \text { (II) }\end{array}$ & 15 & 7 & $\begin{array}{l}A, Y, V, P / M, I, W / F, L, K . \\
D, N, S, T, G, Q, E .\end{array}$ \\
\hline 28. & $\mu$ Bondapak $C_{18}$ & 30 & 4 & Am & $\begin{array}{c}\text { (I) } \mathrm{H}_{2} \mathrm{O}, \mathrm{CH}_{3} \mathrm{OH}, \mathrm{HOAC}, \\
\left(\mathrm{CH}_{3} \mathrm{CO}\right)_{2} \mathrm{O}, \mathrm{pH} 4.1 \\
900: 100: 2.5: 0.05 \\
\text { (II) } \mathrm{H}_{2} \mathrm{O}, \mathrm{CH}_{3} \mathrm{OH}, \mathrm{HOAC} \\
100: 900: 0.25\end{array}$ & $\begin{array}{l}\text { linear } \\
5 \% \text { (II) to } \\
45 \% \text { (II) }\end{array}$ & 20 & 18 & $\begin{array}{l}D, N, S, T, G, H, Q, E, A, R, \\
Y, P, V / M, W, I, F, L, K .\end{array}$ \\
\hline
\end{tabular}

29. Lichrosorb RP8 $30 \quad 3.9 \quad 50 \quad \mathrm{NaOAC} 0.01 \mathrm{M}, \mathrm{CH}_{3} \mathrm{CN}$ isocratic $60 \quad 17 \quad 0, N, S, Q, E, T, G, H, A, R$, $(5 \mu \mathrm{m})$

$\mathrm{pH} 4.6 \quad 75: 25$

$Y, M / P, V, W, I, F, L$.

30. $\mu$ Bondapak $C_{18} \quad 30 \quad 4$

N.G. (I) $\mathrm{NaOAC} 0.01 \mathrm{M}, \mathrm{CH}_{3} \mathrm{OH}$

pH $4.09: 1$

$N, D, T, H, G, Q, E, A, R, T$, $5 \%$ (II) to $S, P, V / M, W, F, I, K, L$.

$\mathrm{pH} 4.0 \quad 1: 9$

\begin{tabular}{|c|c|c|c|c|c|c|c|c|c|}
\hline \multirow[t]{2}{*}{31.} & $\begin{array}{l}\text { Hypersil oDS } \\
(5 \mu \mathrm{m})\end{array}$ & 23 & 4.6 & Am & \multirow{2}{*}{$\begin{array}{l}\text { (I) } \mathrm{NaOAC} 0.01 \mathrm{M} \text { in } \mathrm{H}_{2} \mathrm{O} \\
\text { (II) } \mathrm{NaOAC} 0.01 \mathrm{M} \text { in } \\
\mathrm{CH}_{3} \mathrm{OH}\end{array}$} & \multirow{2}{*}{$\begin{array}{l}\text { linear } \\
27 \%(\text { II }) \text { to } \\
65 \% \text { (II) }\end{array}$} & \multirow[t]{2}{*}{45} & \multirow[t]{2}{*}{18} & $\begin{array}{l}D, E, N, S, T, G, Q, A, Y, C, \\
P, M, V, W, F, I, K, L .\end{array}$ \\
\hline & $\begin{array}{l}\text { Spherisorb ODS } \\
(5 \mu \mathrm{m})\end{array}$ & 23 & 4.6 & Am & & & & & $\begin{array}{l}\text { V/M not resolved } \\
\text { on Spherisorb }\end{array}$ \\
\hline 32. & $\begin{array}{l}\text { Lichrosorb RP } 8 \\
(10 \mu \mathrm{m})\end{array}$ & 25 & 4.6 & 35 & $\begin{array}{l}\text { (I) } \mathrm{NaOAC}_{0.0022 \mathrm{M}} 0.00 \\
\mathrm{pH} 4.9 \\
\text { (II) } \mathrm{CH}_{3} \mathrm{OH}\end{array}$ & $\begin{array}{l}\text { double linear } \\
5 \%(\text { II) to } \\
48 \% \text { (II) }\end{array}$ & 25 & 16 & $\begin{array}{l}D, N, E, S, T, G, Q, A, Y, H, \\
P, M, V, R, I / K / F, L .\end{array}$ \\
\hline
\end{tabular}

\begin{tabular}{|c|c|c|c|c|c|c|c|c|c|}
\hline 33. & $\mu$ Bondapak $C_{18}$ & 30 & 3.9 & 23 & $\begin{array}{l}\text { (I) } \mathrm{NaOAC} 0.005 \mathrm{MH} 5.6 \\
20 \% \mathrm{CH}_{3} \mathrm{OH} \\
\text { (II) } 38 \% \mathrm{CH}_{3} \mathrm{OH} \text { in } \mathrm{H}_{2} \mathrm{O}\end{array}$ & $\begin{array}{l}\text { two step } \\
\text { isocratic } \\
\text { programme } \\
\text { (I) then (II) }\end{array}$ & 45 & 15 & $\begin{array}{l}D, E, N, S, T, G, Q, A, Y, P, \\
V, W, F, L, K .\end{array}$ \\
\hline
\end{tabular}


S. E. Godtfredsen \& R. W. A. Oliver: HPLC of PTH-amino acids

\begin{tabular}{|c|c|c|c|c|c|c|c|c|c|}
\hline \multirow[b]{2}{*}{$\begin{array}{l}\text { Ref. } \\
\text { No. }\end{array}$} & \multicolumn{4}{|c|}{ COLUMN } & \multicolumn{2}{|l|}{ ELUTION } & \multicolumn{3}{|c|}{ SEPARATION } \\
\hline & Support & $\begin{array}{l}1 \\
\mathrm{~cm}\end{array}$ & $\begin{array}{l}\text { i.d. } \\
\text { m.m. }\end{array}$ & $\begin{array}{l}{ }^{\theta} \\
{ }^{\circ} \mathrm{C} \\
\end{array}$ & $\begin{array}{l}\text { Eluant } \\
\text { Composition }(v / v)\end{array}$ & Gradient & $\operatorname{mins}^{t}$ & $N$ & $\begin{array}{l}\text { Assignment (in } \\
\text { order of elution) }\end{array}$ \\
\hline 34. & uBondapak $C_{18}$ & 30 & 4 & 20 & $\begin{array}{l}\text { (I) } 8.0 \mathrm{mM} \mathrm{DETA} \\
20 \mathrm{mM} \mathrm{TCA} \mathrm{pH} 4.2 \\
\text { (II) as for (I) plus } \\
60 \% \mathrm{CH}_{3} \mathrm{CN}\end{array}$ & $\begin{array}{l}\text { concave } \\
5 \%(\text { II }) \text { to } \\
75 \%(I I)\end{array}$ & 36 & 17 & $\begin{array}{l}D, N, S, T, G, H, N, E, A, \\
R, Y, P / V, M, I, L .\end{array}$ \\
\hline 35. & $\begin{array}{l}\text { Spherisorb ODS } \\
(5 \mu \mathrm{m})\end{array}$ & 25 & 4.6 & 37 & $\begin{array}{l}\text { (I) } \mathrm{LIOAC} 0.02 \mathrm{M} \text { pH } 5.2 \\
\text { (II) } \mathrm{LiOAC} 0.1 \mathrm{M} 20 \% \\
\mathrm{CH}_{3} \mathrm{CN} \quad 80 \% \\
\text { pH } 5.2\end{array}$ & $\begin{array}{l}\text { mixed I inear } \\
\text { plus isocratic } \\
25 \% \text { (II) to } \\
95 \% \text { (II) }\end{array}$ & 18 & 19 & $\begin{array}{l}D, G, N, S, T, Q, G, H, A, Y, \\
R, V, M, P, I, W, F, L, K .\end{array}$ \\
\hline
\end{tabular}

\footnotetext{
1 is column length, i.d. is internal diameter, $\theta$ is temperature of the column, $N$ is number of peaks, Am is ambient, N.G. is not given, $t$ is time required for the separation, * calculated from retention data given for PTH-amino acids, DETA is diethylenetriamine, ICA is trichloroacetic acid.
}

\section{REFERENCES TO TABLE}

1. Graffeo, A. P., A. HaAG \& B. L. Karger: Anal. Letters 6. 505-5II (1973)

2. Zimmerman, C. L.. J. J. Pisano \& E. Appella: Biochem. Biophys. Res. Comm. 55, 1220-1224 (1973)

3. Frank, G. \& W. Strubert: Chromatographia 6. 522-4 (1973)

4. HaAG, A. \& K. Langer: Chromatographia 7. 659-622 (1974)

5. Fankhauser, P., P. Fries. P. Stahala \& M. BrenNer: Helv. Chim. Acta 57, 271-277 (1974)

6. De Vries, J. X., R. Frank \& C. Birr: F. E. B. S. Letts. 55, 65-67 (1975)

7. Matthews, E. W., P. G. H. Byfield \& I. MaClNTYRE: J. Chromatog. 110, 369-373 (1975)

8. Bridgen, J., A. P. Graffeo, B. L. Karger \& M. D. W ATERFIELD: Article on page 140 in $» I n s t r u-$ mentation in Amino Acid Sequence Analysis« Edited by R. N. Perham. Academic Press. London 1975

9. Niece, R. L.: P. 233-240, Proc. 1st Int. Conf. in Solid Phase Methods Protein Sequence Analysis. Pierce Chem. Co. Rockford Ill., I (1975)

10. Downing, M. R. \& K. G. Mann: Anal. Biochem. 74, 298-319 (1976)

11. Zimmerman, C. L., E. Appella \& J. J. Pisano: Anal. Biochem. 75, 77-85 (1976)

12. Lominac, G. D. \& H. S. Kingdon: Archiv. Biochem. Biophys. 173, 320-325 (1976)

13. Rodkey, J. A. \& C. D. Bennetr: Biochem. Biophys. Res. Commun. 72. 1407-1413 (1976)

14. Moo-Pen, W. F., D. L. Jue, K. C. Bechtel, M. H. Johnson \& R. M. Schmidi: J. Biol. Chem. 251, 7557-7562 (1976)
15. Bollet, C. \& M. Caude: J. Chromatog. 121, 323-328 (1976)

16. Bridgen, P., G. A. M. Cross \& J. Bridgen: Nature 263, 613-614 (1976)

17. Zimmerman, C. L., E. Appella \& J. J. Pisano: Anal. Biochem. 77, 569-573 (1977)

18. KikTa, E. J. \& E. GrushKa: J. Chromatog. 135, 367-376 (1977)

19. Fong, G. W. K. \& E. GrushKa: J. Chromatog. 142, 299-309 (1977)

20. Ericsson, L. H., R. D. Wade, J. Gragnon, R. M. McDonald, R. Granberg \& K. A. Walsh: Solid Phase Methods in Protein Sequence Analysis. Inserm. Symposium 5. Ed. A. Previero and M. A. Coletti-Previero 137-142. Elsevier (1977)

21. Devillers-Thiery, A. \& G. Blobel: Anal. Biochem. 87, 66-70 (1978)

22. Klapper, D. G., C. E. Wilde \& J. D. Capra: Anal. Biochem. 85, 126-131 (1978)

23. Fong, G. W. \& E. GrushKa: Anal. Chem. 50, 1154-1161 (1978)

24. Hankapiller, M. W. \& L. E. HoOd: Biochemistry $17,2124-2133$ (1978)

25. Zeeuws, R. \& A. D. Strosberg: F. E. B. S. Letts. 85, 68-72 (1978)

26. Van Beeuman, J., J. van Damme \& J. Deley: F. E. B. S. Letts. 93, 373-376 (1978)

27. Margolies, M. N. \& A. Brauer: J. Chromatog. $148,429-439$ (1978)

28. Bhown, A. J., J. E. Mole, A. Weissenger \& J. C. Bennett: J. Chromatog. 148, 532-535 (1978)

29. abrahamsson, M., K. Gröningsson \& S Castensson: J. Chromatog. 154, 313-317 (1978)

30. Elion, J., M. R. Downing \& K. G. ManN: J. Chromatog. 155, 436-438 (1978) 
31. Oestvol.d, G., E. Jensen \& T. Greibrokk: Medd. Norsk Farm. Selsk. 40. 173-179 (1978)

32. Strickland, M., W. N. Strickland, W. F. Brandt, C. Van Holt, B. Wittmann-Liebold \& A. LehmanN: Eur. J. Biochem. 89, 443-452 (1978)

33. Moo-Pen, W. F. M. H. Johnson, K. C. BeChTel \& D. L. Jue: J. Chromatog. 172, 476480 (1979)

34. Annan, W. D.: J. Chromatog. 173, 194-197 (1979)

35. Moser, P. W. \& E. E. Rickil: J. Chromatog. $176,451-455$ (1979)

application of any general observations drawn from the exercise to the understanding and further improvement of the current analytical situation.

Thus, the conclusion included in the fourth observation above can be readily understood in view of the diverse physico-chemical properties of the PTH-amino acids and the mechanism of the chromatographic processes involved when reverse phase support materials are employed. However, the final observation is difficult to understand since current chromatographic theories (7) leads to the conclusion that micro-bore columns (i.d. $\leq 2 \mathrm{~mm}$ ) should be equally as efficient as large diameter columns. Indeed, these theories form the basis of the design and successfull development of at least one commercial micro-bore chromatographic system (5). Further, some of the separations reported before 1977 actually employed reverse phase support material packed in micro-bore stainless steel tubing (e.g. Table I, paper 2). A closer study of the listed papers published prior to 1977, indicate that the probable origins of the current experimental practice is the claim of WoLF (9) and De Stefano $(1,8)$ that use of larger diameter columns improved HPLC separations because »wall effects« were eliminated. In view of these opposing theoretical and practical claims, and the fact that one reverse phase column separations of the PTH-amino acids employing micro-bore Teflon tubing has been reported (Table I, paper 26), it was decided to reinvestigate whether or not efficient separation of PTH-amino acids can be achieved using columns of reverse phase support material packed in micro-bore stainless steel tubing.

\section{MATERIALS AND METHODS}

\subsection{PTH-amino acids}

A mixture of the PTH derivatives of the following amino acids was dissolved in methanol and kept tightly stopped in a freezer before use. PTH derivatives of alanine (A), aspartic acid (D), glutamic acid $(E)$, phenylalanine $(F)$, glycine $(G)$, isoleucine (I), leucine (L), methionine (M), asparagine $(\mathrm{N})$, proline $(\mathrm{P})$, glutamine $(\mathrm{Q})$, serine $(\mathrm{S})$, threonine $(\mathrm{T})$, valine (V), tryptophan (W), tyrosine (Y), and PTH-( $\varepsilon$-phenylthiocarbamoyl)L-lysine $(\mathrm{K})$ were obtained from the Pierce Chemical Co., Rockford, Illinois, USA. The concentration of each amino acid derivative in the stock solution was $0.2 \mathrm{mg} \cdot \mathrm{ml}^{-1}$.

\subsection{Support materials}

Two batches of reverse phase support material of differing particle size, LiChrosorb RP-18,5 $\mu \mathrm{m}$ and $10 \mu \mathrm{m}$, obtained from the Merck Company, Darmstadt, were employed.

\subsection{Stainless steel tubing}

Stainless steel tubing of four different internal diameters, $4.5,3.0,2.1$ and $1.5 \mathrm{~mm}$, in $25 \mathrm{~cm}$ lengths was obtained from Mikrolaboratoriet, Aarhus, fitted with stainless steel frits and was filled with the support material by the slurry packing technique using a mixture of isopropanol and methanol (7:3) as the solvent phase and a slurry packing apparatus manufactured by Magnus Scientific Instrumentation Ltd., Cheshire, U.K.

\subsection{Solvents}

Acetonitrile, HPLC Grade S, was obtained from Rathburn Chemicals, Peeblesshire. Scotland and was filtered through a micro-pore filter (pore-size $0.5 \mu \mathrm{m}$ ) prior to use. 


\subsection{Chromatographic systems}

A Waters chromatographic system equipped with a variable UV-detector Model 450, a solvent programmer Model 660, a solvent delivery system Model 6000A, an injector system Model U6K and a Hewlett Packard integrator Model $3380 \mathrm{~A}$ was used in the first series of experiments.

A Hewlett Packard liquid chromatograph Model 1074 A equipped with a fixed wavelength detector Model 1036 A $(\lambda=254 \mathrm{~nm})$ was employed in the second series of experiments.

\section{RESULTS AND DISCUSSION}

The operating conditions and the average (mean of three consecutive runs) time of appearance for each of the thirteen peaks forming the chromatogram obtained when a mixture of the 17 PTH-amino acids was separated using LiChrosorb RP-18 (10 $\mu \mathrm{m})$, slurry packed in stainless steel tubing of four different diameters and the Waters chromatographic system, is given in Table II.

Before proceeding to discuss the experimental results given in Table II it should be noted that the conditions adopted for the separation of the PTH-amino acids on this support material (Lichrosorb RP-18) were based on those reported in the literature (Table I). The initial separation of the PTH-amino acid mixture was performed on the column of the largest diameter $(4.5 \mathrm{~mm})$ and once satisfactory reproducibility of the peak elution times had been achieved $\left[\frac{\mathrm{dt}}{\mathrm{t}} \times 100 \leqslant\right.$ $1.5 \%$ ] no further studies were made in order to improve the separation by altering the eluant gradient programme or the operating temparature since the aim of the experiments was to determine the effect of reducing the diameter of the chromatographic columns. In the subsequent experiments (as detailed in Table II) with the smaller diameter columns only those variables which would be expected to be linearly related to the volume of the column, namely the sample

\section{Table II}

Summary of the elution times and operating conditions for the HPLC separation of a synthetic mixture of seventeen PTH-amino acids performed on a Water's liquid chromatograph employing columns of LiCrosorb RP-18 $(10 \mathrm{Mm})$ packed in $25 \mathrm{~cm}$ lengths of stainless steel tubing of differing internal diameters at ambient temperature.

\begin{tabular}{ccccc}
\hline Column diameter $(\mathrm{mm})$ & 4.5 & 3.0 & 2.1 & 1.5 \\
Sample volume $(\mu \mathrm{ll})$ & 5.0 & 2.2 & 1.0 & $0.5^{*}$ \\
Flow rate (ml/min) & 3.0 & 1.3 & 0.9 & 0.4 \\
Photometer attenuation & 64 & 32 & 24 & 16 \\
\hline \multirow{2}{*}{ Peak no. } & \multicolumn{4}{c}{ Retention time (min) } \\
\hline 1 & 3.93 & 3.11 & 2.46 & 2.23 \\
2 & 5.01 & 3.54 & 2.94 & 2.72 \\
3 & 5.76 & 3.94 & 3.36 & 3.13 \\
4 & 6.22 & 4.88 & 4.07 & 3.76 \\
5 & 7.32 & 5.80 & 4.60 & 4.23 \\
6 & 7.82 & 6.18 & 5.15 & 4.67 \\
7 & 9.14 & 7.27 & 7.72 & 7.08 \\
8 & 13.23 & 10.98 & 12.51 & 11.93 \\
9 & 14.80 & 16.09 & 15.40 & 14.60 \\
10 & 17.04 & 18.18 & 17.86 & 17.60 \\
11 & 19.07 & 20.41 & 20.24 & 21.40 \\
12 & 19.66 & 20.78 & $20.24 \mathrm{sh}$ & $21.70 \mathrm{sh}$ \\
13 & 20.25 & 21.37 & 21.12 & 22.40 \\
\hline
\end{tabular}

Solvent A was $0.01 \mathrm{M} \mathrm{NaOAc}$, $\mathrm{pH} 4.3$. Solvent $\mathrm{B}$ was $50 \% \mathrm{CH}_{3} \mathrm{CN}+50 \%$ A. Eluent programme: $35 \% \mathrm{~B}$ isocratic for 10 mins. ( 8 mins. when $1.5 \mathrm{~mm}$ column was used), gradient (programme 3 ) to $92 \%$ B for 16 mins. sh is shoulder. ${ }^{*} 5 \mu$ l of a 10 fold dilution in solvent B of the stock solution applied. 
loading and eluant flow rate, together with the derived variable - the spectrophotometer attenuation - were altered in accordance to that calculated from the corresponding changes in the dimensions of the columns employed.

It should also be noted that some separate initial experiments were performed on the larger diameter column $(4.5 \mathrm{~mm})$ in order to ascertain the effect of reducing the pre-column volume and the post-column-pre-detector volume in the Water's system employed. For the former, the Water's $2 \mathrm{ml}$ sample loop in the injection system was replaced by a short $(\simeq 10 \mathrm{~cm})$ length of small bore $(0.15 \mathrm{~mm})$ s.s. tubing, a modification which led to the appearance of regularly spaced baseline solvent "peaks" on the chart output. These were eventually traced to the pulsation effects of the piston pumps employed in the eluant system. Clearly the large $(2 \mathrm{ml})$ sample loop in the injection system had been designed to buffer these effects and therefore the chromatographic data given in Table II were obtained using the un-modified sample injection system. However, improved chromatographic resolution was obtained when the normal long $(\simeq 40 \mathrm{~cm})$ piece of s.s. tubing of large i.d. $(\simeq 0.5 \mathrm{~mm})$ from the column to the photometer was replaced by a short $(5 \mathrm{~cm})$ piece of small diameter $(0.15 \mathrm{~mm})$ s.s. tubing. This simple modification was the only one made to the Water's apparatus in these series of experiments. A study of the chromatographic data given in Table II shows that practically identical separation of the PTHamino acid mixture is obtained using columns of Lichrosorb RP-18 packed in S.S. tubing of differing internal diameters under the conditions specified in the table. Indeed, the only significant change observed when the micro-bore columns were used is that peaks Nos. 11 and 12, which were partially resolved when larger diameter columns are used, merged to form a single peak with a distinct shoulder. It may thus be concluded from this series of experiments that only a very small loss in chromatographic resolution occurs when separations are performed on columns packed in micro-bore s.s. tubing. Further, it should perhaps be emphasised that in this initial series of experiments little or no attempt was made to improve the separations obtained with the micro-bore columns by »finetuning « the operating conditions.
Since the above conclusion is contrary to the claims which ZimmermanN et al. (Table I, paper 17) put forward based on separations performed at elevated temperatures and on columns containing smaller particle size support material, a second series of experiments were performed using an un-modified Hewlett-Packard liquid chromatograph operated at $48^{\circ} \mathrm{C}$ and columns of Lichrosorb RP- 18 of particle size $5 \mu \mathrm{m}$. The advantages to be gained from the use of this particular chromatograph, in addition to the ability to operate readily at elevated temperatures, include the ease whereby the instrument may be automatically operated so as to vary the gradient elution programme and hence to "finetune s the separation conditions. As before, the initial separation of the PTH-amino acid mixture, was performed on the largest diameter column $(4.5 \mathrm{~mm})$, (c.f. Figure 1A).

When the separation was performed, using essential identical conditions of elution, on the smaller diameter column $(3.0 \mathrm{~mm})$ the chromatogram shown in Figure 1B was obtained.

Comparison of the two chromatograms shown in Figures $1 \mathrm{~A}$ and $\mathrm{IB}$ indicates that a distinctly improved separation of the PTHamino acid mixture was obtained on the smaller diameter column. Thus, the peaks numbered 5 and 6 , and the final peak, in both chromatograms are completely resolved on the $3 \mathrm{~mm}$ column and the shoulder of peak number 13 (Figure 1A) is partially resolved in Figure 1B (peak 13). A total of 15 and 16 peaks are apparent in the chromatograms obtained when using the 4.5 and $3.0 \mathrm{~mm}$ column respectively.

Further, whilst the resolution of the separations were reduced when the two micro-bore columns of i.d. 2.1 and $1.5 \mathrm{~mm}$ were used under operating conditions essentially identical to those employed with the wide-bore columns - compare the chromatograms shown in Figures 1A and $1 B$ with those shown in Figures $1 C$ and $1 D$ - it was again found that the chromatographic resolution achieved by use of the smaller of the two columns $(1.5 \mathrm{~mm})$ was better than that obtained when using the $2.1 \mathrm{~mm}$ diameter column. Thus, comparison of the chromatograms shown in Figure IC and ID shows that an extra chromatographic peak (peak No. 13, Figure 1D) is well resolved on the smaller of these two columns. A total of 12 and 13 peaks 


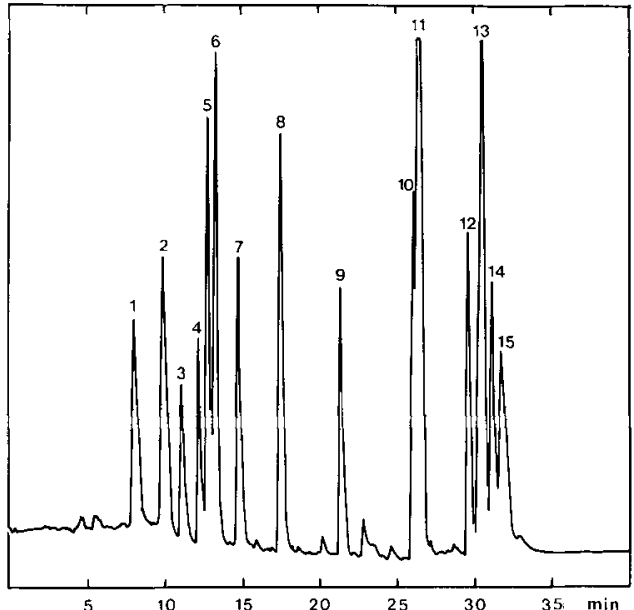

Figure IA. HPLC Chromatogram of PTH-amino acids obtained on a $4.5 \mathrm{~mm}$ (i.d.) column of LiChrosorb RP-18, $5 \mu \mathrm{m}$.

$10 \mu \mathrm{l}$ of the PTH-amino acids dissolved in methanol was injected onto the column. Solvent A was $0.01 \mathrm{~m}-\mathrm{NaOAc}, \mathrm{pH} 4.3$ and solvent $\mathrm{B}$ acetonitrile. Eluent programme: $9.2 \% \mathrm{~B}$ isocratic for 8 mins, linear gradient to $15 \%$ B for 2 mins, linear gradient to $30 \% \mathrm{~B}$ for 15 mins, linear gradient to $35 \%$ B for $1 \mathrm{~min}$, linear gradient to $40 \%$ B for 10 mins. The temperature of the column was $48^{\circ} \mathrm{C}$, the flow was $3.0 \mathrm{ml} \cdot \mathrm{min}^{-1}$ and the chromatogram was monitored at $254 \mathrm{~nm}$. By co-chromatography with authentic specimens the following assignment of the peaks was made: $1=\mathrm{D}, 2=\mathrm{N}, 3=\mathrm{S}, 4=\mathrm{T}, 5=$ Q. $6=\mathrm{G}, 7=\mathrm{E}, 8=\mathrm{A}, 9=\mathrm{Y}, 10=\mathrm{P} .11=\mathrm{M}$ and $\mathrm{V}, 12=\mathrm{W}, 13=\mathrm{I}$ and $\mathrm{F}, 14=\mathrm{L}, 15=\mathrm{K}$. Ordinate in all chromatograms shown is absorbance at $254 \mathrm{~nm}$. The photometer attenuation was 64 .

were observed when using the 2.1 and $1.5 \mathrm{~mm}$ columns respectively.

These interesting findings provided the rationale for the final series of experiments which were again performed on the un-modified Hewlett-Packard Liquid Chromatograph. In these, a systematic study was made of the effect of controlled changes in the elution conditions on the chromatogram obtained earlier when the 1.5 $\mathrm{mm}$ diameter column had been used to separate the PTH-amino acids (Figure ID). Visual comparison of the chromatogram corresponding to the optimal separation obtained as a result of these wfine tuning « studies (c.f. Figure 2) with that obtained in the previous experiments on the

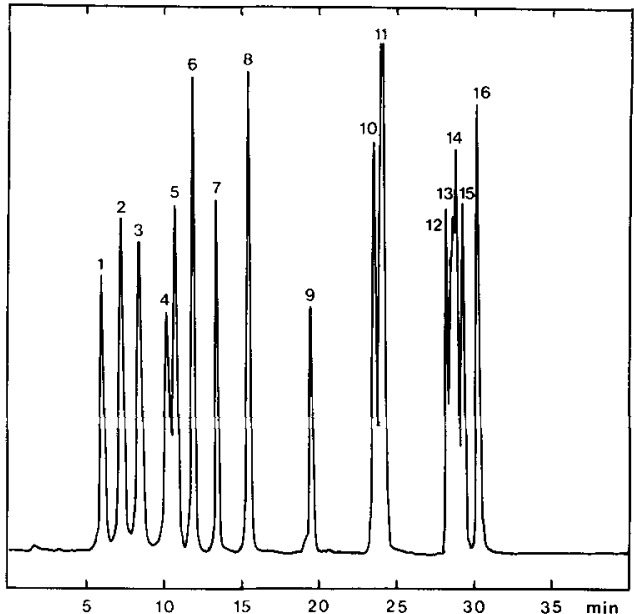

Figure 1B. HPLC Chromatogram of PTH-amino acids obtained on a $3.0 \mathrm{~mm}$ column of LiChrosorb $\mathrm{RP} \cdot 18,5 \mu \mathrm{m}$.

Conditions were as specified by Figure 1A except that $5 \mu \mathrm{l}$ of the solution of PTH-amino acids was applied onto the column which was operated at a flow of $1.5 \mathrm{ml} \cdot \mathrm{min}^{-1}$

same column (Figure 1D) shows that considerable improvements in the chromatographic resolution had been achieved. Thus, peak number 10 in Figure 1D appears as two peaks in

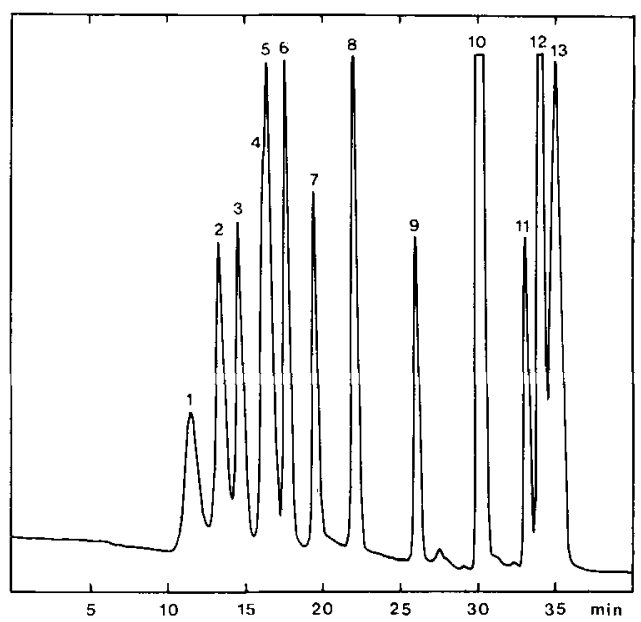

Figure 1C. HPLC Chromatogram of PTH-amino acids obtained on a $2.1 \mathrm{~mm}$ column of LiChrosorb RP-18, $5 \mu \mathrm{m}$.

Conditions were as specified by Figure 1A except that the injection volume was $0.5 \mu l$ and the flow 0.6 $\mathrm{ml} \cdot \mathrm{min}^{-1}$. 


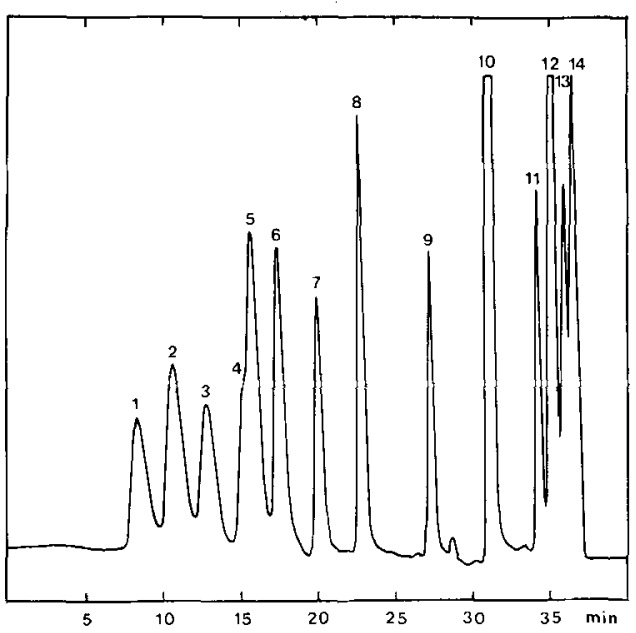

Figure ID. HPLC Chromatogram of PTH-amino acids obtained on a $1.5 \mathrm{~mm}$ column of LiChrosorb RP-18, $5 \mu \mathrm{m}$.

Conditions were as specified by Figure 1A except that the injection volume was $0.2 \mu l$ and the flow 0.5 $\mathrm{ml} \cdot \mathrm{min}^{-1}$.

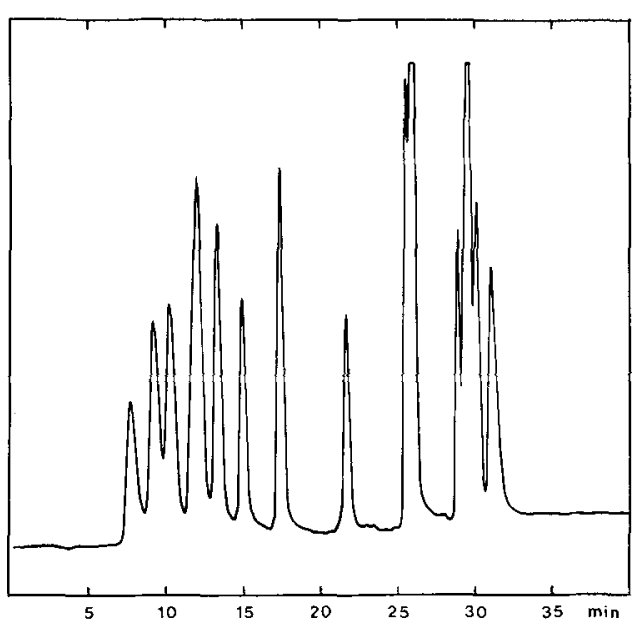

Figure 2. HPLC Chromatogram of PTH-amino acids obtained on a $1.5 \mathrm{~mm}$ column of LiChrosorb RP-18, $5 \mu \mathrm{m}$.

Eluent programme: $10 \%$ B for 2 mins, linear gradient to $15 \%$ B for 4 mins. linear gradient to $30 \%$ B for $15 \mathrm{mins}$, linear gradient to $35 \% \mathrm{~B}$ for $1 \mathrm{~min}$, linear gradient to $40 \% \mathrm{~B}$ for 10 mins, isocratic at $40 \%$ B for 10 mins. Injection volume was $1 \mu l$. Otherwise conditions were as specified under Figure $1 D$.
Figure 2, while the last peak of the chromatograms is only partially resolved in Figure ID, but completely resolved in Figure 2. However, the shoulder on peak number 5 in Figure ID is not apparent in Figure 2.

To summarize the experimental results, comparison of the final chromatogram obtained with the $1.5 \mathrm{~mm}$ i.d. micro-bore column (Figure 2) with the best resolved chromatogram obtained from a wide-bore column (Figure 1B) shows that it is indeed possible to obtain equivalent separations of a complex mixture of PTH-amino acids using columns of Lichrosorb RP-18 $(5 \mu \mathrm{m})$ packed in either wide-bore or micro-bore s.s. tubing. However, before proceeding to comment on the general implications of this particular experimental finding we should like to draw attention to the fact that the precise chromatographic operating conditions for the equivalent separations differ according to the diameter of the column employed (see legends to Figures 1D and 2). This series of experiments demonstrate, therefore, that when the resolution of a chromatographic separation originally performed on a wide-bore column, is reduced when a micro-bore column is used under equivalent conditions, then »fine tuning « of the gradient elution programme may restore the original resolution. This difficulty in directly transferring chromatographic conditions to columns of differing diameter is probably due to the existence of "wall effects «.

The ability of columns of suitable support material packed in micro-bore s.s. tubing to separate mixtures of PTH-amino acids is of practical importance primarily because smaller samples may be analysed. In all of the present experiments the sample volume per $\mathrm{mm}^{2}$ crosssectional area of the column employed was constant, and since the flow rate was correspondingly reduced an effective ten-fold increase in sensitivity of the analytical procedure was achieved by transferring the separation from a 4.5 column to a $1.5 \mathrm{~mm}$ column. Accordingly, the chromatograms shown in the figures were all obtained using the same photometer attenuation despite of the fact that varying sample volumes were applied. Two further advantages would also accrue from the adoption of micro-bore columns for this particular separation. The first would be that one of the major running costs of the assay, that of the organic solvents acetonitrile 
or methanol commonly employed, could be significantly reduced because of the reduced flow rates employed. Secondly, the amount of expensive column support material would also be significantly reduced; by a factor of nine if the column diameters employed were changed from $4.5 \mathrm{~mm}$ to $1.5 \mathrm{~mm}$ as in these studies.

The adoption of micro-bore columns could theoretically also lead to an economical chromatographic system in which complete separation of each of the 17 components in the mixture of PTH-amino acids was achieved by using longer lengths of s.s. tubing to form longer columns possessing correspondingly greater resolving power. More importantly, the significant reduction in eluant flow rates resulting from the use of micro-bore columns should greatly simplify the as yet unresolved general problem of sin-line coupling of liquid chromatographs to mass spectrometers - a solution to which is necessary before unambiguous identification of the eluted components can be made routinely (3).

Clearly, all of the above arguments in favour of adopting micro-bore S.S. columns for the analysis of PTH-amino acids, and more generally for analyses involving liquid chromatography, apply with even more force to separations performed on columns with even smaller diameter than those employed in these studies. At present time no chromatographic separations of PTH-amino acids performed on sub microbore (i.d. $\leqslant 0.2 \mathrm{~mm}$ ) diameter s.s. columns have been reported but these may be confidently predicted now that several pumps capable of operating at very low flow rates are commercially available and details of a sub micro-litre detector cell has been published (4). To cover the interim period before the results of such studies are published it is recommended, to those using HPLC methods in sequence studies, that serious consideration should be given to the advantages to be gained from the adoption and implementation of the experimental findings presented above.

\section{ACKNOWLEDGEMENTS}

The authors would like to thank Professor M. OTTESEN for his sustained interest and encouragement throughout these studies. In addition we would like to thank Dr. I. SVENDSEN for his practical assistance and for the assignment of the peaks in the chromatogram shown in Figure $1 \mathrm{~A}$ as well as Mrs. H. SøRENSEN for preparing the drawings. We would also like to acknowledge Mrs. E. JENSEN of the library of the Carlsberg Research Center for advice obtained during the course of the bibliographic studies involved in the completion of Table I. Finally, one of us (R.W.A.O.) acknowledges the hospitality of the laboratory during the period in which this work was performed.

\section{REFERENCES}

1. Beachell, H. C. \& J. J. De Stefano: Effect of CSP support particle size on the performance of larger diameter liquid partition columns. J. Chromatogr. Sci. 10, 480-486 (1972)

2. Edman, P.: Determination of the amino acid sequence in peptides. Arch. Biochem. Biophys. 22, 475-476 (1949)

3. Guichon, G. \& P. J. Arpino: Liquid chromatography - mass spectrometry coupling. Anal. Chem. 51, 682A-701A (1979)

4. Hershberger, L. W., J. B. Callis \& G. D. Christian: Sub-microliter flow-through cuvette for fluorescence monitoring of high performance liquid chromatographic. Anal. Chem 51, 14441446 (1979)

5. Ishit, D., K. Asai, K. Hibi, T. JonokuChi \& M. NAGAYA: A study of micro-high-performance liquid chromatography. J. Chromatog. 144, 157-168 (1977)

6. Rosmus, J. \& Z. DeyL: Methods for the identification of $\mathrm{N}$-terminal amino acids in peptides and proteins; part B - PTH-amino acids. J. Chromatog. 70, 292-339 (1972)

7. SCOTT, R. P. W. \& P. KuCERA: Mode of operation and performance characteristics of micro-bore columns for use in liquid chromatography. J. Chromatog. 169, 51-72 (1979)

8. Stefano, J. J. De \& H. C. Beachell: Performance of large diameter liquid partition analytical columns packed with controlled surface porosity support. J. Chromatogr. Sci. 8, 434438 (1970)

9. Wolf, J. P.: Large diameter columns for preparative scale high speed liquid chromatography. Anal. Chem. 45, 1248-1250 (1973) 